Supplement of Atmos. Chem. Phys., 21, 8437-8454, 2021

https://doi.org/10.5194/acp-21-8437-2021-supplement

(C) Author(s) 2021. CC BY 4.0 License.

(c) (i)

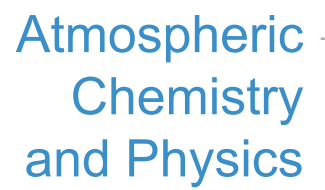

Supplement of

\title{
Modelling the impacts of iodine chemistry on the northern Indian Ocean marine boundary layer
}

Anoop S. Mahajan et al.

Correspondence to: Anoop Mahajan (anoop@tropmet.res.in) and Swaleha Inamdar (swaleha.inamdar@tropmet.res.in)

The copyright of individual parts of the supplement might differ from the article licence. 


\section{Supplement:}

7 Table S1: Monthly means and standard deviations of $\mathrm{O}_{3}, \mathrm{NO}_{2}, \mathrm{NO}, \mathrm{NO}_{3}, \mathrm{OH}, \mathrm{HO}_{2}$ mixing

8 ratios (unit in parenthesis) over the domain region for the model simulations in January,

9 April, and July for the HAL scenario along with the absolute difference and absolute

10 difference percentage between HAL and BASE. The table also includes monthly mean

11 values only over the MBL.

\begin{tabular}{|c|c|c|c|c|c|c|}
\hline & January & April & July & January & April & July \\
\hline & \multicolumn{3}{|c|}{$\mathrm{O}_{3}$ (ppbv) } & \multicolumn{3}{|c|}{$\mathrm{NO}_{3}$ (pptv) } \\
\hline & \multicolumn{6}{|c|}{ Over the whole domain } \\
\hline HAL & $32.16 \pm 9.76$ & $29.64 \pm 10.79$ & $23.34 \pm 8.85$ & $7.64 \pm 8.08$ & $10.38 \pm 15.53$ & $4.52 \pm 6.14$ \\
\hline HAL-BASE & $1.25 \pm 0.69$ & $0.98 \pm 0.69$ & $0.21 \pm 0.22$ & $0.46 \pm 0.35$ & $0.50 \pm 0.74$ & $0.16 \pm 0.25$ \\
\hline \multirow[t]{2}{*}{$\begin{array}{c}\text { HAL-BASE } \\
\%\end{array}$} & $3.75 \pm 3.17$ & $3.21 \pm 3.58$ & $0.89 \pm 1.04$ & $5.73 \pm 13.6$ & $4.68 \pm 9.78$ & $3.52 \pm 6.84$ \\
\hline & \multicolumn{6}{|c|}{ Only over the MBL } \\
\hline HAL & $28.17 \pm 7.83$ & $24.17 \pm 6.42$ & $19.49 \pm 5.97$ & $4.47 \pm 5.44$ & $2.99 \pm 4.09$ & $2.38 \pm 3.94$ \\
\hline HAL-BASE & $1.31 \pm 0.66$ & $1.22 \pm 0.65$ & $0.15 \pm 0.18$ & $0.45 \pm 0.31$ & $0.29 \pm 0.29$ & $0.11 \pm 0.17$ \\
\hline \multirow[t]{3}{*}{$\begin{array}{c}\text { HAL-BASE } \\
\% \\
\end{array}$} & $4.45 \pm 3.37$ & $4.80 \pm 3.49$ & $0.77 \pm 1.13$ & $9.20 \pm 14.15$ & $8.81 \pm 10.08$ & $4.55 \pm 7.47$ \\
\hline & \multicolumn{3}{|c|}{$\mathrm{NO}_{2}$ (ppbv) } & \multicolumn{3}{|c|}{ OH (pptv) } \\
\hline & \multicolumn{6}{|c|}{ Over the whole domain } \\
\hline HAL & $0.43 \pm 1.27$ & $0.30 \pm 0.77$ & $0.27 \pm 0.79$ & $0.14 \pm 0.05$ & $0.26 \pm 0.07$ & $0.28 \pm 0.08$ \\
\hline HAL-BASE & $0.008 \pm 0.019$ & $0.007 \pm 0.018$ & $0.004 \pm 0.012$ & $0.004 \pm 0.004$ & $0.008 \pm 0.005$ & $0.005 \pm 0.004$ \\
\hline $\begin{array}{c}\text { HAL-BASE } \\
\% \\
\end{array}$ & $1.89 \pm 10.63$ & $2.15 \pm 5.71$ & $1.63 \pm 5.33$ & $3.29 \pm 3.14$ & $3.02 \pm 1.94$ & $1.82 \pm 1.40$ \\
\hline & \multicolumn{6}{|c|}{ Only over the MBL } \\
\hline HAL & $0.10 \pm 0.46$ & $0.06 \pm 0.30$ & $0.07 \pm 0.29$ & $0.15 \pm 0.05$ & $0.27 \pm 0.08$ & $0.27 \pm 0.08$ \\
\hline
\end{tabular}




\begin{tabular}{|c|c|c|c|c|c|c|}
\hline HAL-BASE & $0.004 \pm 0.007$ & $0.002 \pm 0.007$ & $0.002 \pm 0.006$ & $0.005 \pm 0.004$ & $0.008 \pm 0.005$ & $0.005 \pm 0.004$ \\
\hline $\begin{array}{c}\text { HAL-BASE } \\
\%\end{array}$ & $3.47 \pm 11.25$ & $3.51 \pm 6.08$ & $2.52 \pm 5.95$ & $3.64 \pm 3.39$ & $3.05 \pm 1.89$ & $1.75 \pm 1.36$ \\
\hline & \multicolumn{7}{|c|}{ NO (pptv) } \\
\hline & \multicolumn{7}{|c|}{ Over the whole domain } \\
\hline HAL & $49.49 \pm 221.23$ & $36.66 \pm 164.95$ & $38.79 \pm 173.78$ & $7.10 \pm 1.49$ & $10.18 \pm 1.64$ & $9.24 \pm 1.97$ \\
\hline HAL-BASE & $1.60 \pm 5.61$ & $1.57 \pm 6.33$ & $1.03 \pm 4.32$ & $0.51 \pm 0.39$ & $0.44 \pm 0.27$ & $0.21 \pm 0.14$ \\
\hline $\begin{array}{c}\text { HAL-BASE } \\
\text { \% }\end{array}$ & $3.22 \pm 14.32$ & $4.36 \pm 8.15$ & $2.64 \pm 6.46$ & $6.76 \pm 4.87$ & $4.16 \pm 3.09$ & $2.18 \pm 1.52$ \\
\hline \multicolumn{7}{|c|}{ Only over the MBL } \\
\hline HAL & $12.56 \pm 85.76$ & $10.38 \pm 77.48$ & $11.64 \pm 58.45$ & $7.32 \pm 1.12$ & $9.8 \pm 1.36$ & $8.67 \pm 1.53$ \\
\hline HAL-BASE & $0.70 \pm 2.78$ & $0.72 \pm 2.78$ & $0.45 \pm 1.95$ & $0.67 \pm 0.36$ & $0.53 \pm 0.25$ & $0.23 \pm 0.14$ \\
\hline $\begin{array}{c}\text { HAL-BASE } \\
\text { \% }\end{array}$ & $5.48 \pm 14.86$ & $7.07 \pm 8.58$ & $3.76 \pm 7.15$ & $8.38 \pm 4.51$ & $5.17 \pm 2.98$ & $2.63 \pm 1.49$ \\
\hline
\end{tabular}

12

13 Figures:
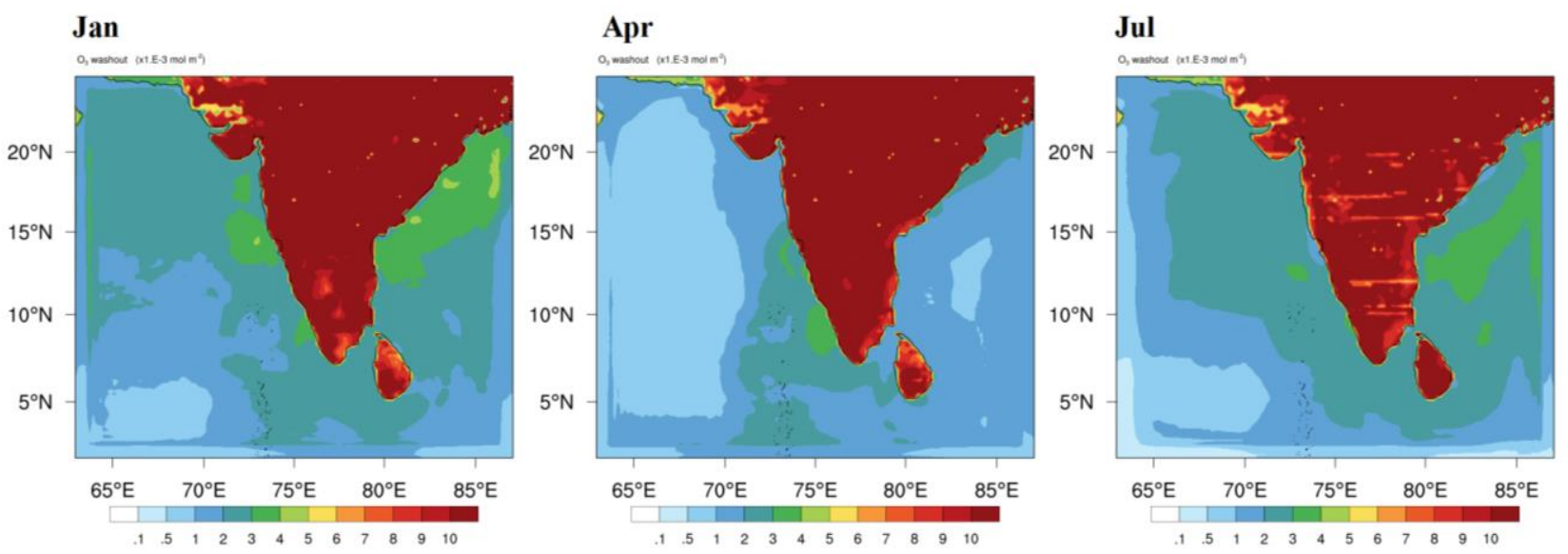

14

15 Figure S1: Simulated $\mathrm{O}_{3}$ washout $\left(\mathrm{x}^{-3} 0^{-3} \mathrm{~mol} \mathrm{~m}^{-2}\right)$ in HAL scenario in three months.

16 

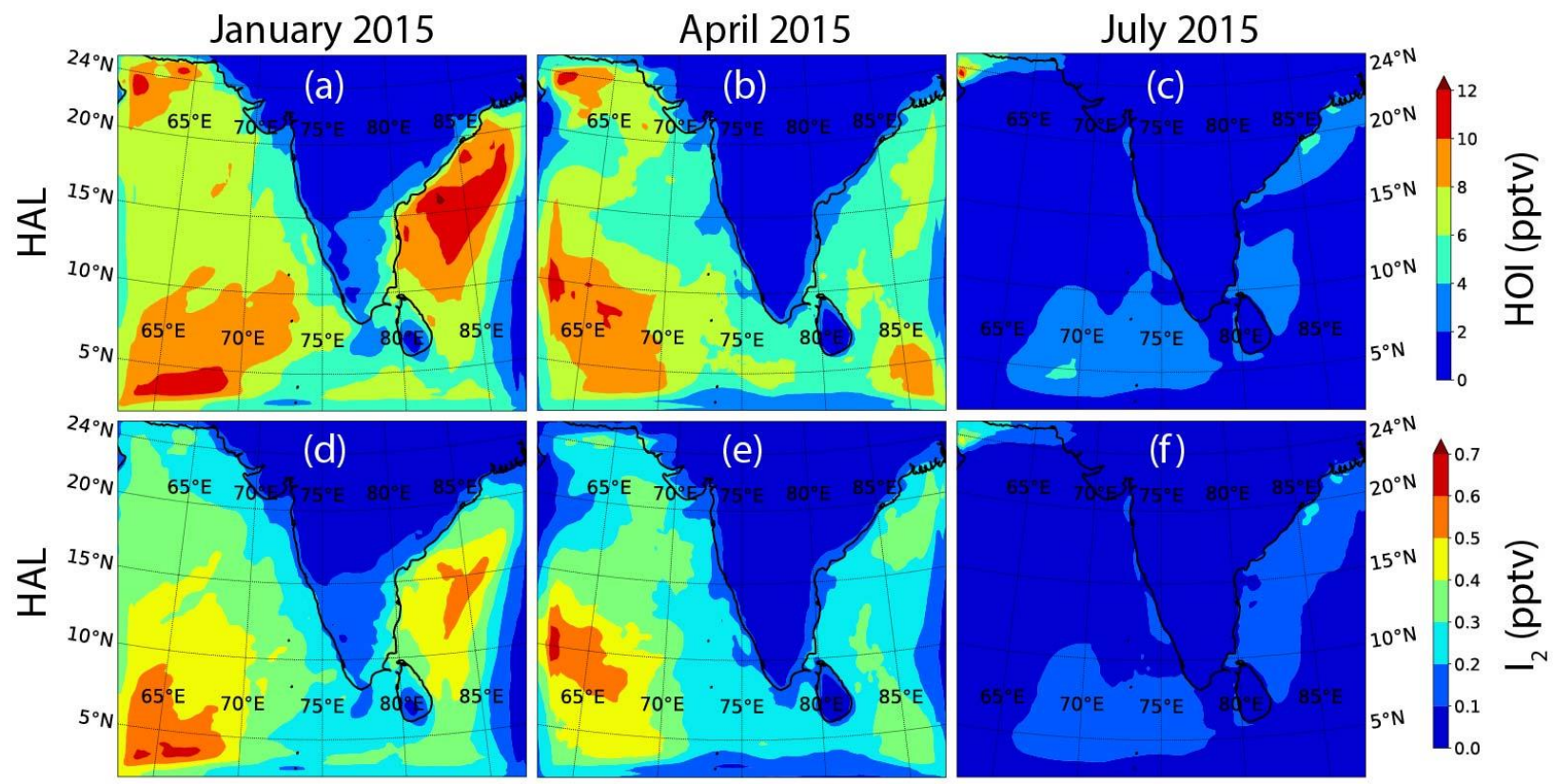

18 Figure S2: Seasonal variability in the HOI and $\mathrm{I}_{2}$ mixing ratios indicate that the emissions of HOI

19 and $\mathrm{I}_{2}$ are lowest during July, coinciding with the lowest $\mathrm{O}_{3}$ mixing ratios.

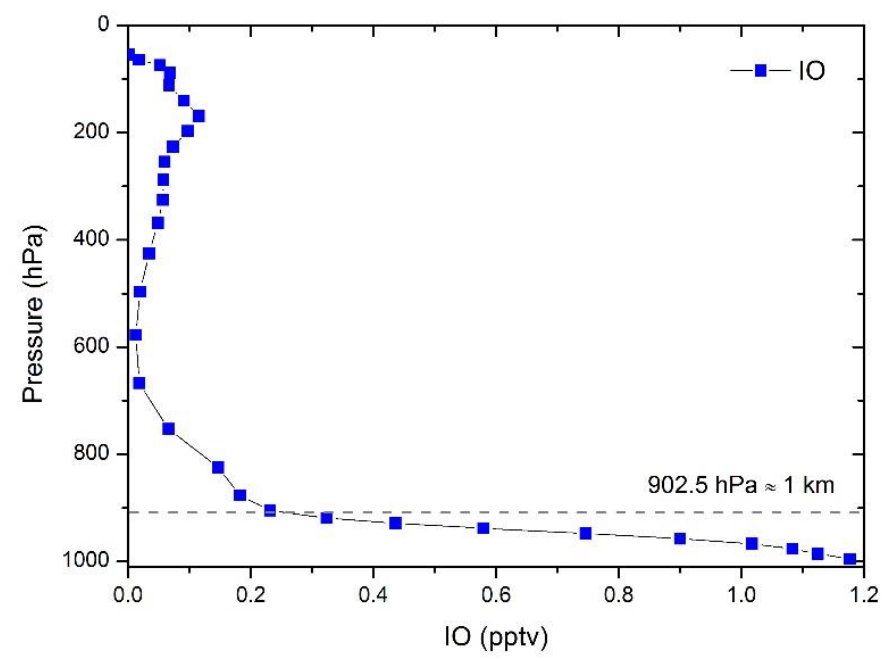

21 Figure S3: The vertical distribution of IO in the WRF-Chem simulations is shown. The dashed

22 line indicates the assumed boundary layer height below which the column is integrated for all the

23 other figures in the manuscript. 